\title{
Duodenal displacement and acquired intrathoracic stomach in an extreme premature baby on jejunostomy feeds- a case report
}

\author{
Kailas P Bhandarkar*, Catherine A Richards
}

Evelina London Children's Hospital, London UK

*Corresponding Author: Kailas P Bhandarkar, Evelina London Children's Hospital, London, UK.

Received date: November 11, 2020; Accepted date: December 30, 2020; Published date: January 05,2021

Citation: Kailas P Bhandarkar, Catherine A Richards. (2021) Duodenal displacement and acquired intrathoracic stomach in an extreme premature baby on jejunostomy feeds- a case report. International Journal of Clinical Case Reports and Reviews. 6(1); DOI: 10.31579/2690-4861/094

Copyright: (C) 2021 Kailas P Bhandarkar, This is an open-access article distributed under the terms of the Creative Commons Attribution License, which permits unrestricted use, distribution, and reproduction in any medium, provided the original author and source are credited.

\section{Summary}

Repeated and persistent displacement forces can lead to acquired abnormalities in the position of the foregut in premature neonates. Cow milk protein intolerance should be considered early as a cause of feed intolerance, recurrent vomiting and other gastrointestinal symptoms in premature neonates. Upper gastrointestinal contrast study cannot be used to diagnose gastro-oesophageal reflux disease.

\section{Background}

An extremely premature neonate developed abnormal position of the stomach and duodenum secondary to prolonged nasojejunal tube feeds. This led to acquired intrathoracic stomach which required surgical correction. She was diagnosed to have Cow milk protein intolerance and improved with hydrolysed feeds. Postoperatively she progressed well and is currently on a mixture of oral and gastric feeds.
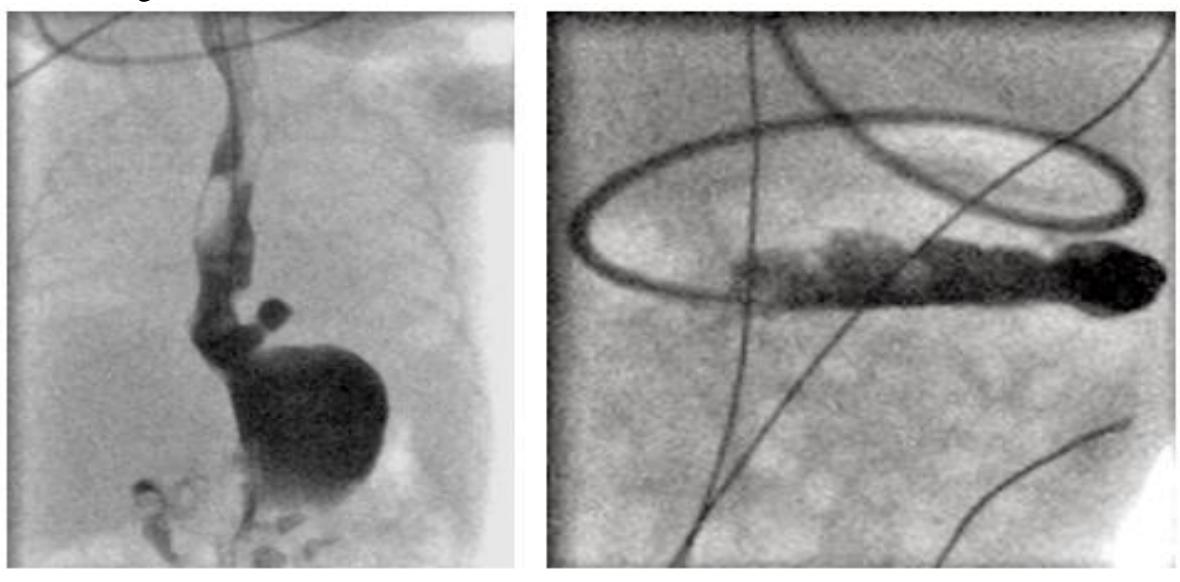

Figure: $1(\mathrm{a})$

Figure: 1(a) and (b): Contrast study showing moderate sized "hiatus hernia". Gastric contrast has ascended into the oesophagus (the baby subsequently vomited). Normal anatomy of the duodenal loop and duodeno-jejunal flexure with oro-jejunal feeding tube in situ.

However, feed intolerance with bilious vomiting persisted, despite jejunal tube feeds. At 17 weeks of age (corrected to term gestational age),

\section{Case report}

We report an extremely premature neonate born at $23+5$ weeks of gestation weighing 620 grams. She was commenced on nasogastric tube feeds which were initially maternal expressed breast milk; subsequently the feeds were transitioned to standard preterm formula. However, she failed to tolerate these feeds and had persistent bile stained vomits and aspirates.

At 6 weeks of age (29+3 weeks corrected gestational age), an upper GI contrast study was performed. It showed a moderately sized hiatus hernia which filled promptly with contrast and marked gastro-oesophageal reflux to the level of the cervical oesophagus, followed by vomiting (Fig. 1a). Duodenal anatomy was normal. Therefore, an oro-jejunal feeding tube was passed (Fig. 1b). she developed hematochezia and a plain abdominal x-ray showed extensive pneumatosis (Fig. 2). She remained otherwise well, and symptoms resolved promptly with cessation of feeds. She was diagnosed to have Cow milk protein intolerance (CMPI) and was commenced on a hydrolyzed formula via her jejunal tube. At week later, she was tolerating her target volume of hydrolyzed formula by jejunal tube. She showed improvement on her growth curves and was weaned off parenteral 
nutrition. 4 weeks later (at $45+2$ weeks corrected gestational age), a repeat upper Gastrointestinal contrast study demonstrated the entire stomach to be in the chest and duodenal loop displaced caudally and medially (Fig. 3)

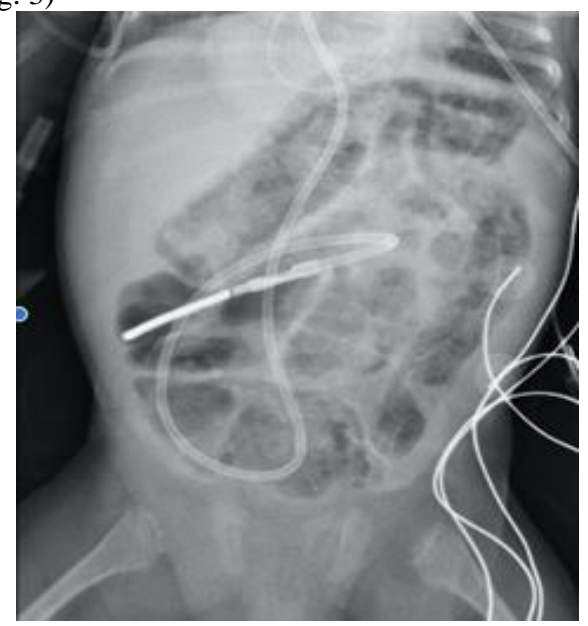

Figure 2: Plain abdominal film shows widespread pneumatosis. (Also apparent is the nasogastric tube sitting in the thorax and the transpyloric jejunal tube shows displacement of the duodenum).

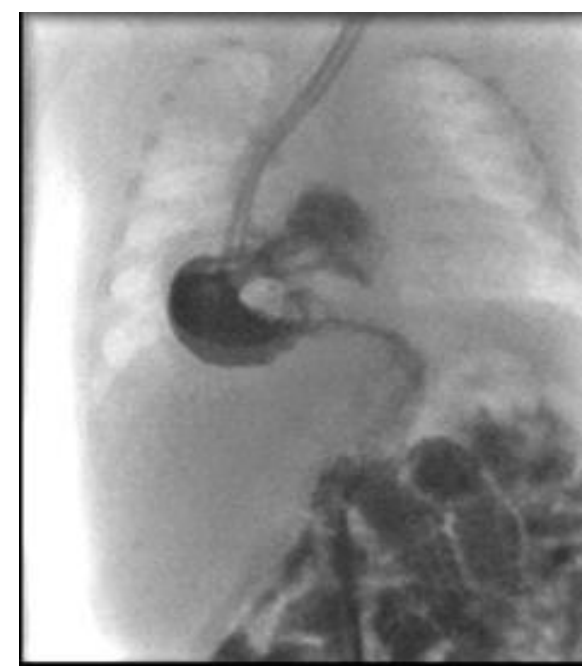

Figure 3: Contrast study showing that the whole stomach has herniated into the thorax. The duodenal anatomy is distorted with caudal and medial displacement. The duodeno-jejunal flexure is not clearly identified (oral transpyloric jejunal tube in place).

\section{Differential diagnosis}

The differential diagnosis here includes Cow milk protein intolerance, overfeeding, Gastro-oesophageal reflux, Hiatus hernia and malrotation of the midgut.

\section{Management}

A decision was made for surgery to restore the stomach into the abdomen to permit gastric feeds in future. At 24 weeks of age (48 weeks corrected gestational age), she had a laparotomy. The entire stomach was in the thorax but was readily brought down into the abdomen without any tension. The wide hiatus was repaired. A Stamm gastrostomy was formed that served both to fix the stomach in the abdomen and for passage of trans-pyloric jejunal extension for feeds. As suggested by preoperative imaging, the duodenum was very mobile and displaced medially. However, the remaining small bowel mesentery was normal. Malrotation was not present.

\section{Outcome and follow up}

She promptly re-established jejunal feeds postoperatively. She is currently 3 years old and is tolerating both oral solids and gastric bolus feeds.

\section{Discussion:}

Forces generated during vomiting- The force causing movements of gastric contents during gastroesophageal reflux is the relatively small pressure gradient normally between abdomen and thorax generated by respiration (7-22cmH2O) (Fig. 4a). In contrast to gastroesophageal reflux, activation of the emetic reflex leads to retching and true vomiting, which generate much more powerful forces. In addition, activation of emetic reflex is accompanied by a giant retrograde contraction within the small intestine that drives intestinal contents and bile back into the stomach. During retching, the anterior abdominal wall muscles and entire diaphragm contract rhythmically and synchronously, with negative pressure pulses in the thorax mirrored by positive pressure pulses in the abdomen. This progressively builds up a substantial trans-diaphragmatic pressure gradient $(200-300 \mathrm{mmHg}$ in the cat). During retching, there is a pulsing cephalad displacement of the gastroesophageal junction, with the fundus of the stomach drawn through the diaphragmatic hiatus into the thorax [1] (Fig. 4b). This culminates in vomiting, achieved by the somatic muscles of the anterior abdominal wall compressing the relaxed stomach. After vomiting has taken place, tissue elasticity normally restores the stomach to the normal intra-abdominal position [2]. However, in this premature neonate with flimsy connective tissue, the repeated retching forces resulted in permanent and progressive displacement of the stomach (Fig 4c, 1a). The combination of a stiff transpyloric feeding tube and gastric displacement resulted in stretching of the duodenal attachments with medial and caudal displacement of the duodenum. 

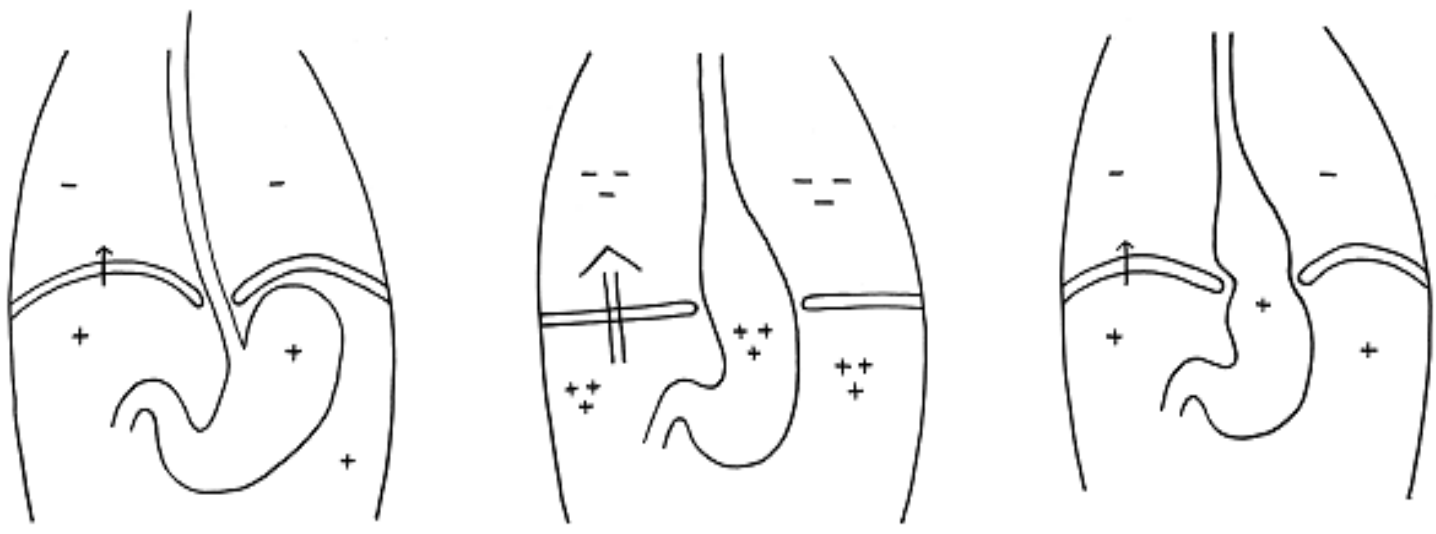

Figure 4 a, b, c: Diagrammatic representation of abdomino-thoracic pressure gradients arising as a result of forceful contractions of the diaphragm and anterior abdominal wall during retching. a,Small pressure gradient (small arrow) during quiet respiration. b,Large abdomino-thoracic pressure gradient (large arrow) during forceful diaphragmatic contraction (flattened diaphragm) drives the gastroesophageal junction through the diaphragmatic hiatus into the thorax creating a physiological "hiatus hernia". c,Normally gastric position is restored when vomiting ceases but in this neonate with flimsy connective tissue, the stomach remained displaced within the thorax

Diagnostic caveats: Our patient exhibited symptoms related to feeds that were due to CMPI but were initially attributed to other conditions. CMPI may cause vomiting symptoms in infants which is often mistaken for gastroesophageal reflux disease [3]. Failure to respond to simple anti-reflux measures or the presence of atypical symptoms (such as bile-stained vomiting and aspirates in our neonate) should lead to reappraisal of the diagnosis. CMPI may cause pneumatosis and blood in the stool which may be mistaken for necrotising enterocolitis in neonates. Our neonate had extensive pneumatosis but was otherwise well and responded promptly to withdrawal of cow milk from her diet. A contrast study cannot be used to diagnose reflux disease. A contrast study does not distinguish physiologic and pathologic reflux [3]. Moreover, a contrast study of vomiting due to activation of the emetic reflex will show the appearance of a hiatus hernia (which in this situation is physiological and temporary) and gastric contents entering the oesophagus, and maybe misinterpreted as reflux [2].

\section{Summary}

Repeated/persistent displacement forces can lead to acquired abnormalities in the position of the foregut in premature neonates. Cow milk protein intolerance should be considered early as a cause of feed intolerance, recurrent vomiting and other gastrointestinal symptoms in premature neonates. Upper gastrointestinal contrast study cannot be used to diagnose gastroesophageal reflux disease.

\section{References.}

1. McCarthy LE, Borison HL. (1974) Vomiting: radiographic and oscillographic correlates in the decerebrate cat. Gastroenterology.67:1126-1130.

2. Richards CA, Does retching matter? Reviewing the evidence-Physiology and forces, J Pediatr Surg.

3. Rosen R, Vandenplas $\mathrm{Y}$ et al. (2018) Pediatric Gastroesophageal Reflux Clinical Practice Guidelines: Joint Recommendations of the North American Society for Pediatric Gastroenterology, Hepatology, and Nutrition and the European Society for Pediatric Gastroenterology, Hepatology, and Nutrition JPGN Volume 66(3). 